\title{
Generative and Modular Simulation Models for Supply and Manufacturing Networks
}

\author{
Pavel Gocev, Tim Hellfeuer \\ Siemens Energy, Huttenstraße 12, 10553 Berlin, Germany; \\ pavel.gocev@siemens.com, tim.hellfeuer@siemens.com
}

\section{Introduction}

The application of Discrete-Event Simulation (DES) models for purposes of planning and optimization of factories and supply networks is characterized with various abstraction levels and granularities of the model structure. These two aspects are dependent on the complexity of the systems to be simulated, the business goals to be achieved and the project objectives where the simulation models are deployed. This is especially intensified when different product parts and components on different levels withn the supply networks are included into one model, like production lines and work centers within existing and emerging factory shop floors combined with the network of suppliers and additionally flavoured with the ramp up of new products, new work centers or both. $V$ ery often the complexity is increased due to the organizational nature of production types and different project groups with own modelling paradigms. This is particularly a characteristic of supply networks that deliver very complex commodity products like whole power plants or respective components.

The usual foundation to describe and model such complex systems is the data around the three principal consisting domains (PPR): Prockucts to be delivered (raw-materials, parts, components, finished products), Proceses that produce them (from supply chain steps down to operational steps) and Resources necessary to acomplish the work (suppliers, factories, production lines, work centers, machines, etc.). $Y$ et the data is not enough to build the simulation model that, following the paradigm of digital twin, also represents its behaviour as well as the interdependencies between the consisting elements within the PPR-D omains. These interactions, behaviours, and cause-and-effect graphs are usually embodied as a procedural programming, affecting the scope and the depth of the modelled logic and therewith they influence the abstraction levels within the model. The situation is even more complex, in a case when the simulation models represent a workshop-like production and the same simulation model is intended to be deployed for various factories and different products within one big and multifaceted company like Siemens Energy. In opposite of the typical assembly lines like in automotive or electronics industry, here we are talking about product and respective parts and components that are running through different resources in an arbitrary sequence defined by product features and manufactruing technologies available in the considered factories or within the supply network.

\section{Model Structure}

W hit this paper we present a kind of shell for Generative and Modular Simulation Models for Supply and $M$ anufacturing Networks realized in Tecnomatix Plant Simulation by Siemens. The solution bears on paradigm of data driven model generation and scenario definition, as well as modular structure that offers a flexible and scalable deployment on arbitrary granularity levels with defined but extendable building blocks. The focus here is put on the data that describe the main PPR-Domains as well as scenario parameters, which variation offers twofold controls within the simulation modle. On one side their values can be directly alternated and on the other side for each scenario they change a certain behavioural logic within the model through adjustment of some modules that are constating part of the model. During the sumulation experiment the operationala data is acquired and at the end of each simulation experiment the statistical data is calculated and prepared for external analysis.

\section{Modules}

The solution is built on modules mainly embodied in 
the Plant Simulation own language SimT alk, that are interlinked through their communication and every module takes a decision that shapes and controls the behavourals during the simulation experiment.

The first aspect of the platform are the modules for data ingestion (DIM) and model generation (M GM). The external PPR-D ata is imported into the Simualtion M odel Shell through available interfaces of Tecnomatix Plant Simulation and stored in the tables within or in a sharedmemory database SQL ite. The structures of data and the simulation model follow some standards like ISA-95 resp. IEC 62264 which yilds is a precondition for flexible and scalable solutions. The specific and executable simulation model is generated in seconds, that offers the user an execution of arbitrary simulation experiments.

The Production Orders M odule (POM) generates the necessary production orders based on the information from the Bill of Materials and the Disposition Module (DM ) governs their dispatching in the supply chain and shop floor. Every Production O rder has assigned at least one or more Routings that are structured around the information within the Bill of Processes. The M ake or B uy Module (MBM) decides if a Production Order will be produced internally or externally at suppliers. The internal production (make) is controlled by the Scheduling M odule (SM) that is founded on one of the ideas of Industrie 4.0 where for each Process the Products and Resources are communicating and take decisions regarding the context situation. Here within each Process the main function assigns a specific Production Order to a certain Resource from the alternative list considering the situation within the shop floor and the most suitable prioritization logic. The processing of the Products is monitored by the $M$ anufacturing $M$ odule ( $M M$ ) that regulates functionalities like: setting up of Ressources, single part processing, batching, batch splitting, assembly, disassembly as well as an initiation for movement of the parts which is carry out by the Transportation M odule (TM). As the Resources are the foundation of the supply and manufactuirn networks, the Resource M odule (RM) regulates the planned and stochastic events that influence their availability and therewith a numerous behaviours and decisions during the simulation experiment. Firstly, every Resource has assigned one or more Resource Life Cycle States like commissioning, ramp-up, fully allocated, ramp-down, in-relocation, etc. Within every state the Resources are further constrained with the shift model that defines working hours, breaks, shift handover, weekends and holidays. The last limitations are the presumable outages and failures that are stochsticly described for each Resource. The Simulation A nalytics Module (SAM) is the crucial element that moitors the objects, acquires data, calculates some Key Performance Indicators and prepares the results for further external analysis.

A dittionaly there are some modules for model analysis, monitoring, optimization. Some of these modules are neither used during the business simulation experiment nor by the user but rather by developers for model improvement purposes.

\section{Simulation Experiments and Results}

One set of PPR-D ata and defined scenario parameters are necessary input for performing a simulation experiment. The preformance of the simualtion model respectivly the sumulation time is dependat on a scenario complexity and the modules and features that are activated in a particular scenario. This posibilities to define each scneario through parameters, enables the user to carry out different experiments that can include: a combination of a long term planing with the short-time scheduling, an involvement or ignorance of the suppliers, definition of make or buy constraints, an integration of factory planing aspects like ramp-up, a relocation and ramp-down, an introduction of al ternating shift models or a variation of prioritization rules.

The operational data gatherd during the simulation experiment is a foundation for further analysis and evaluation. At the end of each experiment the usual production and logistcs K ey Performance Indicators are calculated and displayed to the user for further analysis to support the definition of new scenarios or their comparison as well as take actions in the real factory if necessary.

\section{Outlook}

The very complex and modulary structured shell for generation of simulation models offers a various possibility for further devleopments in scope and in depth. The current modules have been developed to rerspond on the demands within the Siemens Energy organization and emerged on the base of business requirements. Additional developments are possible and necessary to cover other requirements and functionalities that are not still covered and implemented. 